\title{
Rapid Changes in Light Scattering from Human Polymorphonuclear Leukocytes Exposed to Chemoattractants \\ Discrete Responses Correlated with Chemotactic and Secretory Functions
}

Itzhak Yuli and Ralph Snyderman Laboratory of Immune Effector Function, Howard Hughes Medical Institute and Division of Rheumatic and Genetic Diseases, Department of Medicine, Duke University Medical Center, Durham, North Carolina 27710

bstract. A platelet aggregometer was adapted for the simultaneous measurement of perpendicular light scattering in addition to light transmission. The addition of chemoattractants to polymorphonuclear leukocyte suspensions evoked a single wave of increased light transmission, whereas the perpendicular scattering measurement demonstrated a previously unrecognized biphasic response. The first perpendicular scattering response had no detectable latency and peaked at $10 \pm 1 \mathrm{~s}$, then decayed rapidly. The second response peaked at $40 \pm 5 \mathrm{~s}$, and decayed over several minutes. The dose-response curve of

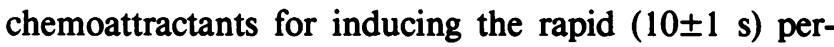
pendicular scattering peak corresponded to that which initiated chemotaxis. Initiation of the slow $(40 \pm 5 \mathrm{~s})$ peak required 10-fold higher amounts of chemoattractants, and the dose-response curve correlated with the induction of lysosomal enzyme secretion and superoxide anion production. Low doses of aliphatic alcohols, which have been shown to enhance chemotaxis but to inhibit secretion and superoxide anion production, abolished the slow perpendicular light-scattering response but left the fast response intact. Stimulants of secretion induced only slow and prolonged responses that were best observed in transmission measurements. In an attempt to resolve the origin of the light-scattering responses, the morphological

\footnotetext{
Address reprint requests to Dr. Snyderman. 1984.

Received for publication 1 July 1983 and in revised form 12 January
}

J. Clin. Invest.

(c) The American Society for Clinical Investigation, Inc. 0021-9738/84/05/1408/09 $\$ 1.00$

Volume 73, May 1984, 1408-1417 changes of polymorphonuclear leukocytes were examined microscopically. Neither aggregation nor morphological whole cell polarization could be correlated with changes in light transmission or perpendicular scattering, which suggested that the source of scattering is of subcellular dimensions.

The rapid perpendicular light-scattering response of polymorphonuclear leukocytes to chemoattractants appears to record an initial event in the stimulus-response coupling, and its measurement should provide a useful new tool for the study of leukocyte function. The biphasic nature of the light-scattering responses to chemoattractants, moreover, correlates with the dual regulation of the chemotactic and secretory responses of leukocytes.

\section{Introduction}

Chemotaxis is a dynamic process that requires the migrating cells to identify a chemoattractant and to perceive its concentration distribution. Regardless of the gradient perception mechanism, be it spacial or temporal (1), the study of stimulusresponse coupling of the chemoattractant receptor should, when possible, be approached kinetically. Attempts were therefore made to monitor the time course of morphological polarization of polymorphonuclear leukocytes (PMN) ${ }^{1}$ from round to the wedged shape that is characteristic of migrating cells (2-5). This change in morphology, termed polarization, which appears to be a requisite step for a chemotactic response, begins within seconds after exposure of leukocytes to chemoattractants in suspension (6). It has previously been shown that PMN sus-

\footnotetext{
1. Abbreviations used in this paper: $\mathrm{CB}$, cytochalasin B; fMet-Leu-Phe, $N$-formyl-methionyl-leucyl-phenylalanine; $\mathrm{EC}_{50}$, half-effective concentration; HHB, Hank's/Hepes buffer; PMA, 4 $\beta$-phorbol $12 \alpha$-myristateacetate; PMN, polymorphonuclear leukocytes.
} 
pensions exposed to chemoattractants in a platelet aggregometer demonstrate a rapid increase in transmitted light $(7,8)$. By analogy to platelet aggregation, the major light transmission response of PMN has been attributed to cellular aggregation (9). By the same analogy the aggregation of PMN was anticipated to be preceded by changes in whole cell shape $(10,11)$. We therefore hoped to monitor the kinetics of PMN polarization by studying the initial changes in light transmission following the addition of chemoattractants to stirred suspensions of the cells. However, reproducible measurements of changes in light transmission necessitated the use of a dense cell suspension $(\leq 5$ $\left.\times 10^{6} \mathrm{PMN} / \mathrm{ml}\right)(12,13)$. These turbid suspensions compel the transmitted light to undergo multiple scattering, since it is unlikely for a light beam scattered by one cell not to be further scattered by additional cells before encountering the detector. Multiple scattering distorts the proportionality between the magnitude of change in scattered light and the actual fraction of responding cells, which are responsible for the changes. This lack of proportionality is most prominent with transmitted light (i.e., forward scattering in the incident light direction), but drops sharply with the observation angle. In an attempt to better quantify the light response, an additional perpendicular measurement was introduced. The responses of PMN to chemoattractants were then characterized by quantifying simultaneous forward and perpendicular light scattering. The addition of the perpendicular measurements revealed information not previously appreciated by recordings of light transmission alone. Moreover, the perpendicular light measurement allows quantitative analysis of rapid PMN responses to chemoattractants and provides a new tool for studying the relationship between chemoattractant receptor occupancy and discrete cellular responses.

\section{Methods}

Reagents. $\quad N$-formyl-methionyl-leucyl-phenylalanine (fMet-Leu-Phe), cytochalasin B (CB), Hepes and $4 \beta$-phorbol $12 \alpha$-myristate $13 \alpha$-acetate (PMA) were purchased from Sigma Chemical Co. (St. Louis, MO). Colchicine was obtained from Eli Lilly \& Co. (Indianapolis, IN). Dextran T-500 was purchased from Pharmacia Fine Chemicals (Piscataway, NJ).

Cell preparation. Heparinized venous blood $(10 \mathrm{U} / \mathrm{ml}$; Upjohn Co., Kalamazoo, MI) was obtained from healthy donors, mixed 1:1 (v/v) with 3\% Dextran T-500 (wt/vol), and allowed to sediment at room temperature for $25 \mathrm{~min}$. The supernatant was centrifuged on a FicollHypaque density gradient according to Boyum (14). The sedimented cells were exposed to $0.2 \% \mathrm{NaCl}$ for $35 \mathrm{~s}$ at room temperature to lyse the remaining erythrocytes. After isotonicity was restored by adding an equal volume of $1.6 \% \mathrm{NaCl}$, the suspension was centrifuged at $300 \mathrm{~g}$ for $10 \mathrm{~min}$ at $4^{\circ} \mathrm{C}$. The isolated PMN were washed and resuspended in Hank's balanced salt solution (Gibco Laboratories, Grand Island, $\mathrm{NY}$ ) and supplemented with $10 \mathrm{mM}$ Hepes at $\mathrm{pH} 7.2$ (Hank's/Hepes buffer [HHB]). Platelet contamination did not exceed $2 \%$ (cell number ratio).

Light-scattering kinetics. PMN samples $(0.4 \mathrm{ml})$ containing $10^{7}$ cells/ $\mathrm{ml} \mathrm{HHB}$ were measured in $1-\mathrm{ml}$ cuvettes at $37^{\circ} \mathrm{C}$ with a dual aggregation meter (DP-247E; Sienco, Inc., Morrison, CO) that had been modified to monitor simultaneously transmitted and perpendicularly scattered red light $(\lambda=670 \pm 20 \mathrm{~nm})$. The geometrical constraints and the angular response of the BES photodiod detector (VTB-113; Vactec Inc., Maryland Heights, MO) confined the light signal to $\pm 10^{\circ}$. Changes in light intensity were displayed on a dual pen recorder (Sienco, Inc.). The stirring of all samples was adjusted to produce a steady base line for at least $4 \mathrm{~min}$ before any experimental manipulation. Stimulation was achieved by a rapid addition of $\leq 10 \mu \mathrm{l}$ ( $\leq 2.5 \% \mathrm{vol}$ increase) of the appropriate stimulant with a 25- $\mu$ l microsyringe (Hamilton Co., Reno, NV) that was fixed to avoid any interference with the magnetic stirring bar. Mixing lag time was shown to be $<1 \mathrm{~s}$ in high speed recording measurements. The baseline light intensity varied among experiments due to variations in the system's extinction efficiency, which originated predominantly from differences amongst donors and the optical properties of the cuvettes (Sienco, Inc.). The transmission and the perpendicular scattering base lines were therefore set independently as reference values at the initiation of each measurement. The units of the light intensity of the transmission response were taken directly from the recorder chart scale (i.e., arbitrary scale). The perpendicular scattering readings were calibrated to the same arbitrary scale by multiplying the chart scale by the ratio of amplification of the transmission to perpendicular scattering signals. The contribution of the dilution effect due to the addition of the stimulant was subtracted from the reported light responses by using a calibration tracing of buffer addition for each experimental setting. Signals were considered significant $(P<0.01)$ when their net amplitude, i.e., after subtracting of the dilution artifact, shifted from base line by more than twice the base-line noise range. The latter was estimated as four standard deviation ranges based on the fact that the base lines are continuous measurements. The short time course of back scattering (i.e., $>90^{\circ}$ ) was monitored using PMN samples of $3 \mathrm{ml}$ containing $10^{7}$ cells $/ \mathrm{ml} \mathrm{HHB}$ measured at $37^{\circ} \mathrm{C}$ with a light-scattering monophotometer (Model 6000, C. N. Wood Mfg. Co., Newtown, PA) aligned to a single pen recorder. Excitation was achieved by an He-Ne laser ( $\lambda=6,328 \AA$; Laser Products, San Marcos, CA). Measurements were taken for $\leq 5 \mathrm{~min}$, during which time reference systems maintained constant output intensities.

Scanning electron and phase-light microscopy. Samples $(0.4 \mathrm{ml})$ of $10^{7} \mathrm{PMN} / \mathrm{ml}$ were stimulated with fMet-Leu-Phe and monitored for transmission and perpendicular scattering response during various incubation periods. The response to the chemoattractant was terminated by a rapid addition of $0.4 \mathrm{ml}$ ice-cold buffered (pH 7.2) $10 \%$ formalin (6). The samples were centrifuged at $400 \mathrm{~g}$ for $15 \mathrm{~min}$ and the pellets were prepared for scanning electron microscopy by critical point drying (15). When samples were examined by phase-light microscopy $(\times 400)$, they were removed from the aggregometer at the same times indicated above, placed directly on a microscope slide, and immediately observed.

\section{Results}

Effect of PMN concentration on forward and perpendicular light scattering. The characteristics of the light scattered from PMN exposed to a chemoattractant were derived from measurements of transmission and perpendicular scattering using various cell densities and $10 \mathrm{nM}$ fMet-Leu-Phe, a dose that is optimal for inducing chemotaxis (16). Fig. 1 represents a series of coupled measurements of transmission and perpendicular scattering that were recorded simultaneously from a single cell suspension for each PMN density tested. The transmission measurement demonstrates the well known alterations in light transmission associated with PMN exposed to a chemoattractant. The mea- 


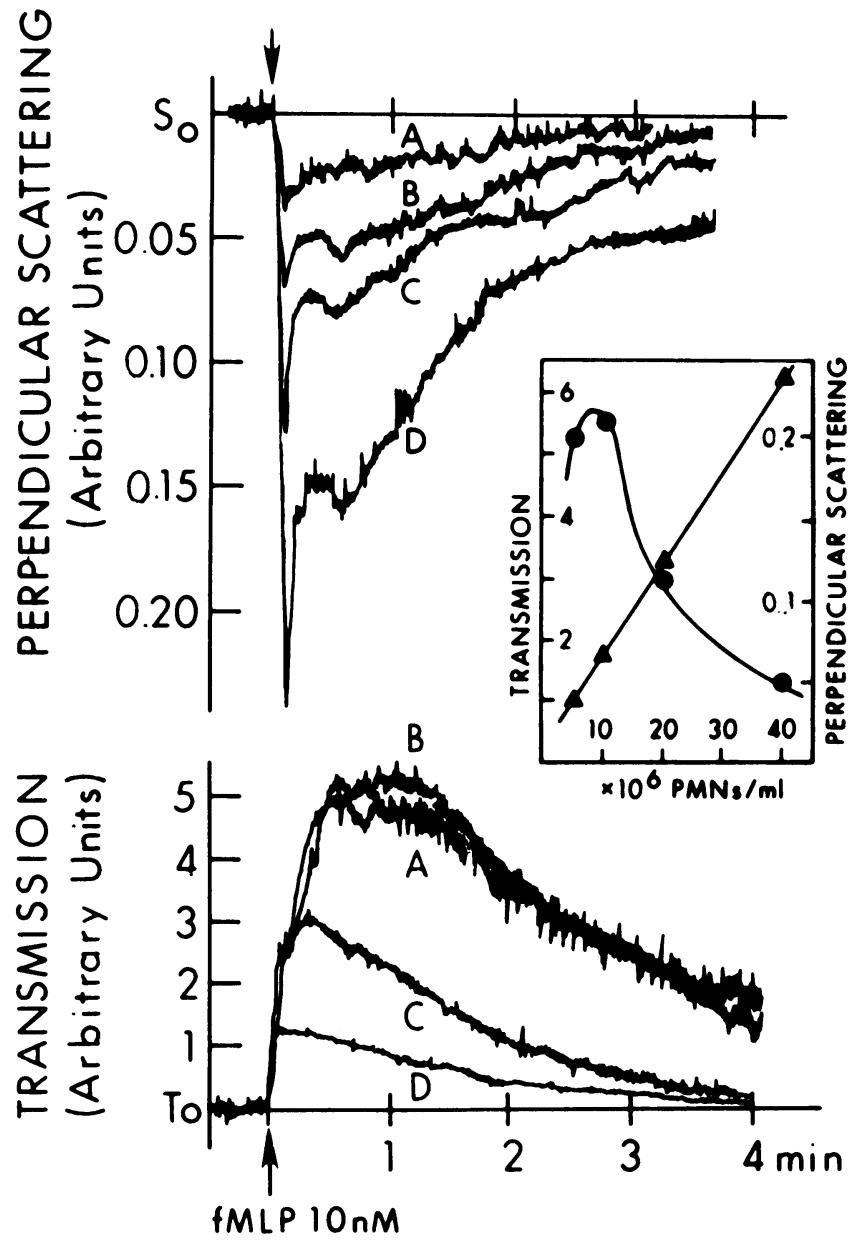

Figure 1. Effect of cell density on simultaneous transmission and perpendicular scattering obtained from PMN suspensions in response to fMet-Leu-Phe. PMN densities were adjusted to 5- $(A), 10-(B), 20-$ $(C)$, and 40-million $(D)$ cells/ml HHB. fMet-Leu-Phe was added to a final concentration of $10 \mathrm{nM}$ at the arrow-indicated zero-time points only after arbitrary transmission (To) and perpendicular scattering (So) base lines had been established. The units of the shifts in light intensity, though arbitrary, were matched according to the aggregation meter and recorder adjustments. The inserted panel summarizes the dependence of the maximal amplitudes of the transmission taken between 10 and $60 \mathrm{~s}$ from stimulation ( $\bullet$ ), and the rapid perpendicular scattering all taken at $10 \mathrm{~s}$ from stimulation $(\Delta)$ on the cell densities.

surements reveal increased transmission intensity manifested by a single complex reversible wave pattern $(7,8)$. In contrast, the chemoattractant induced a decrease in the intensity of the perpendicular scattered light. This decrease began concomitantly with the increase in the transmission intensity. However, at least two easily distinguishable transient responses were observed in the perpendicular measurement. The first was a sharp, highly synchronized response that peaked at $10 \pm 1$ s (determined by high-speed recording; not shown) and decayed symmetrically after $\sim 20 \mathrm{~s}$ into a slower response. The latter response showed a less synchronized pattern that peaked at $\sim 40 \mathrm{~s}$ and decayed gradually over several minutes. The primarily reciprocal nature of the light measurements recorded at the forward and the perpendicular angles clearly indicates that the phenomenon observed resulted from changes in light scattering rather than light absorption. Absorption would be characterized by a proportional decrease of the light intensity at both observation angles.

In response to the chemoattractant stimulation, the amplitudes of the transmission patterns reached a maximal level at $\sim 10^{7} \mathrm{PMN} / \mathrm{ml}$ and declined sharply for cell densities above 2 $\times 10^{7} \mathrm{PMN} / \mathrm{ml}$. In contrast, the amplitude of the perpendicular scattering response was linear with PMN density throughout the experimental range tested, with a highly significant linear correlation ( $r=0.9975$ ) of the rapid response. The maximum change in transmission light intensity following stimulation of $10^{7} \mathrm{ml}$ PMN ranges between 2 and $6 \%$ of the base-line unstimulated level. This broad range of the relative response reflects the variance in both the base lines and the responses amongst donors. Yet, the responses were found to be significantly different from the base-line level by $t$ test $(P<0.01, n=6)$. In the perpendicular measurements, the change in light intensity reached $14-19 \%$ of base-line level with a higher significance of the rapid response $(P<0.001, n=7)$. Note however that in the transmission mode, circa 300 times more light was recorded than in the perpendicular mode. In other words, the reduction in the perpendicular scattering intensity covers only circa $1.5 \%$ of the increased transmission response.

The relatively small changes in light transmission responses to chemoattractant stimulation of PMN necessitates experimental systems of considerable PMN density in order to reach a sufficient signal to noise ratio. In turn, these conditions yielded multiple scattering, a notion that is supported by the high optical densities of the PMN suspensions and their lack of linearity with the cell numbers. The optical densities of unstimulated PMN suspensions were $0.95 \pm 0.10,1.28 \pm 0.10,1.62 \pm 0.20$, and $1.95 \pm 0.16$ (mean \pm SD) OD units at $670 \mathrm{~nm}$ for 5-, 10-, 20-, and 40-million $\mathrm{PMN} / \mathrm{ml}$, respectively.

Low angle measurements were obscured by the light dispersion of the incident beam to a cone of $\pm 12^{\circ}$ at $10^{7} \mathrm{PMN} /$ $\mathrm{ml}$ and an optical path of $1 \mathrm{~cm}$. Therefore, we could not monitor the kinetics of the light response to chemoattractants at low angle diffraction. Yet, we found no detectable angular dependence of the light response around the minimum scattering intensity angle of $115^{\circ}$ in the range between 90 and $140^{\circ}$. All chemoattractant-response patterns made at these angles of observation were mutually indistinguishable and resembled the perpendicular scattering responses (data not shown).

Effects of chemoattractant dose on light-scattering responses. The effects of fMet-Leu-Phe dose on the light-scattering responses of $10^{7} \mathrm{PMN} / \mathrm{ml}$ is depicted in Fig. 2. By comparing light transmission with perpendicular scattering, one can appreciate the resolution between rapid and slower responses even in the trans- 


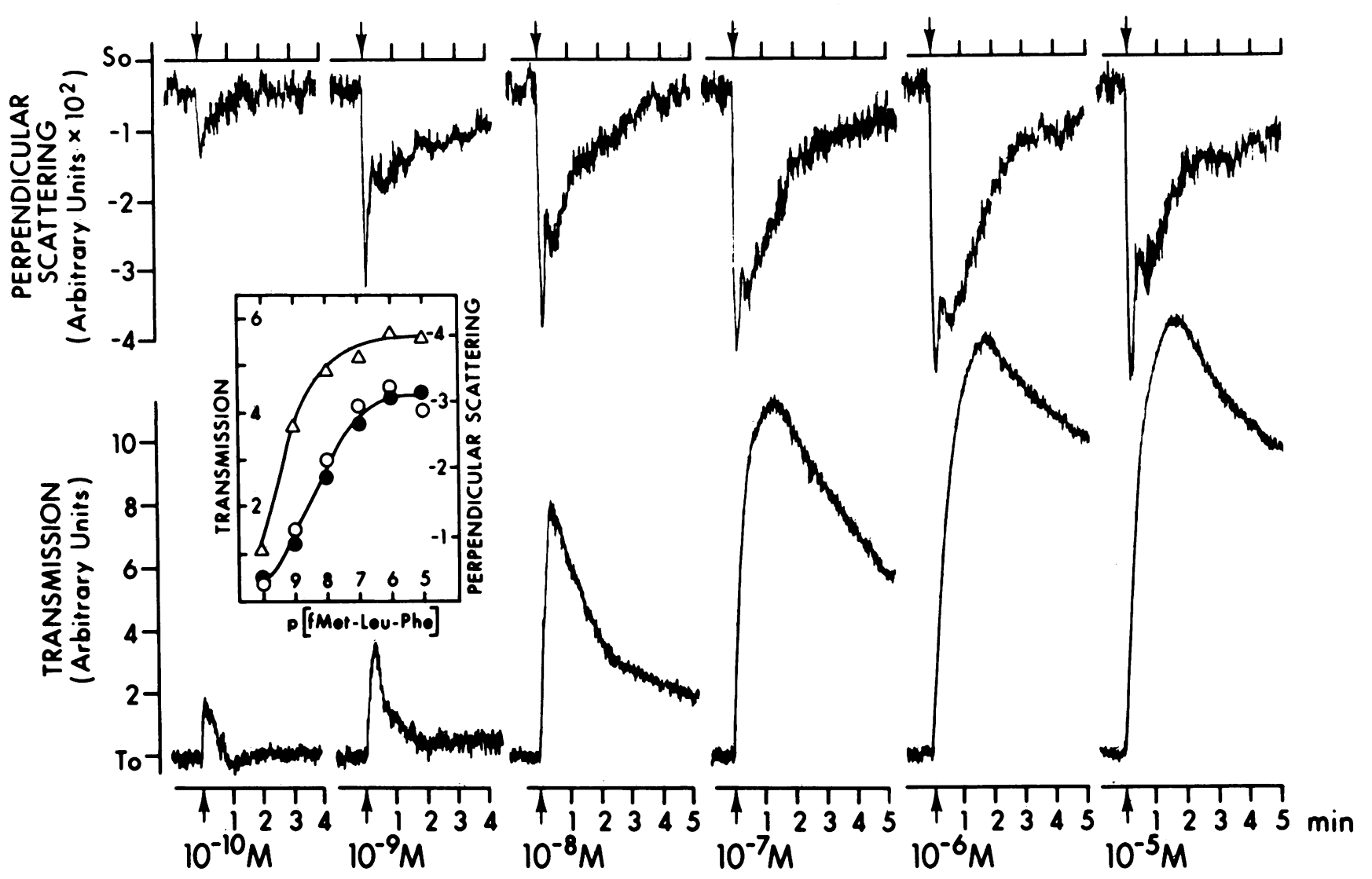

Figure 2. fMet-Leu-Phe dose-dependent transmission and perpendicular scattering responses of PMN suspensions. The simultaneous measurements were carried out with the various fMet-Leu-Phe doses administrated at constant 4- $\mu$ l injections into the systems at zero-time, indicated by the arrows. The inserted panel summarizes the dependence of the maximal amplitudes of the rapid $(\Delta)$ and slow (o) perpendicular scattering and the transmission $(\bullet)$ responses on $\mathrm{fMet}-\mathrm{Leu}-\mathrm{Phe}$ concentration.

mission measurement. At very low fMet-Leu-Phe concentrations, primarily the rapid response was observed, which is indicated by a transient and sharp symmetrical deflection. As the fMet-Leu-Phe concentration was raised, the slower response became visible in the perpendicular scattering, and could also be seen in the forward scattering, as judged by the transmission decay. Examination of the peak responses demonstrated that the transmission and the slow perpendicular response amplitudes increased linearly with dose up to $1 \mu \mathrm{M}$ fMet-Leu-Phe and plateaued thereafter. In contrast, the fast peak amplitude increased abruptly with dose up to $10 \mathrm{nM}$ fMet-Leu-Phe, beyond which the increases were reduced by far fewer. Assuming that the slow scattering response begins instantaneously upon the introduction of the chemoattractant (see below), its increasing overlap with the fast response at doses above $10 \mathrm{nM}$ fMet-LeuPhe could account for the moderate increase in the fast peak amplitude at the higher dose range. Therefore, assuming that the fast peak approaches maximum at $10 \mathrm{nM}$ fMet-Leu-Phe, a half-effective concentration $\left(\mathrm{EC}_{50}\right)$ of $0.4 \mathrm{nM}$ can be attributed to this response. The $\mathrm{EC}_{50}$ of the slow perpendicular and the transmission responses would range between 3 and $8 \mathrm{nM}$.

To determine if the phenomenon observed using fMet-LeuPhe is common to other chemoattractants, the effects of C5a and the synthetic hexapeptide fNle-Leu-Phe-Nle-Tyr-Lys were also examined. Fig. 3 presents the dose-dependent effects of C5a on the PMN light-scattering response, and reveals patterns analogous to fMet-Leu-Phe. Similar results were also obtained for the hexapeptide (data not shown). For both C5a and the hexapeptide, a distinction of an order of magnitude between the $\mathrm{EC}_{50}$ of the rapid $(0.06 \mathrm{nM})$ and slow $(0.3-0.7 \mathrm{nM})$ responses was observed. This distinction is in agreement with the dose ranges for their induction of chemotaxis and secretion (17).

Demonstration of rapid and slow responses in transmission recordings by modifying the PMN. Evidence for the separation of the light transmission curve into rapid and slow responses were demonstrated in experiments using PMN that had been treated with low concentrations of aliphatic alcohols. It has previously been shown that these alcohol concentrations enhance 


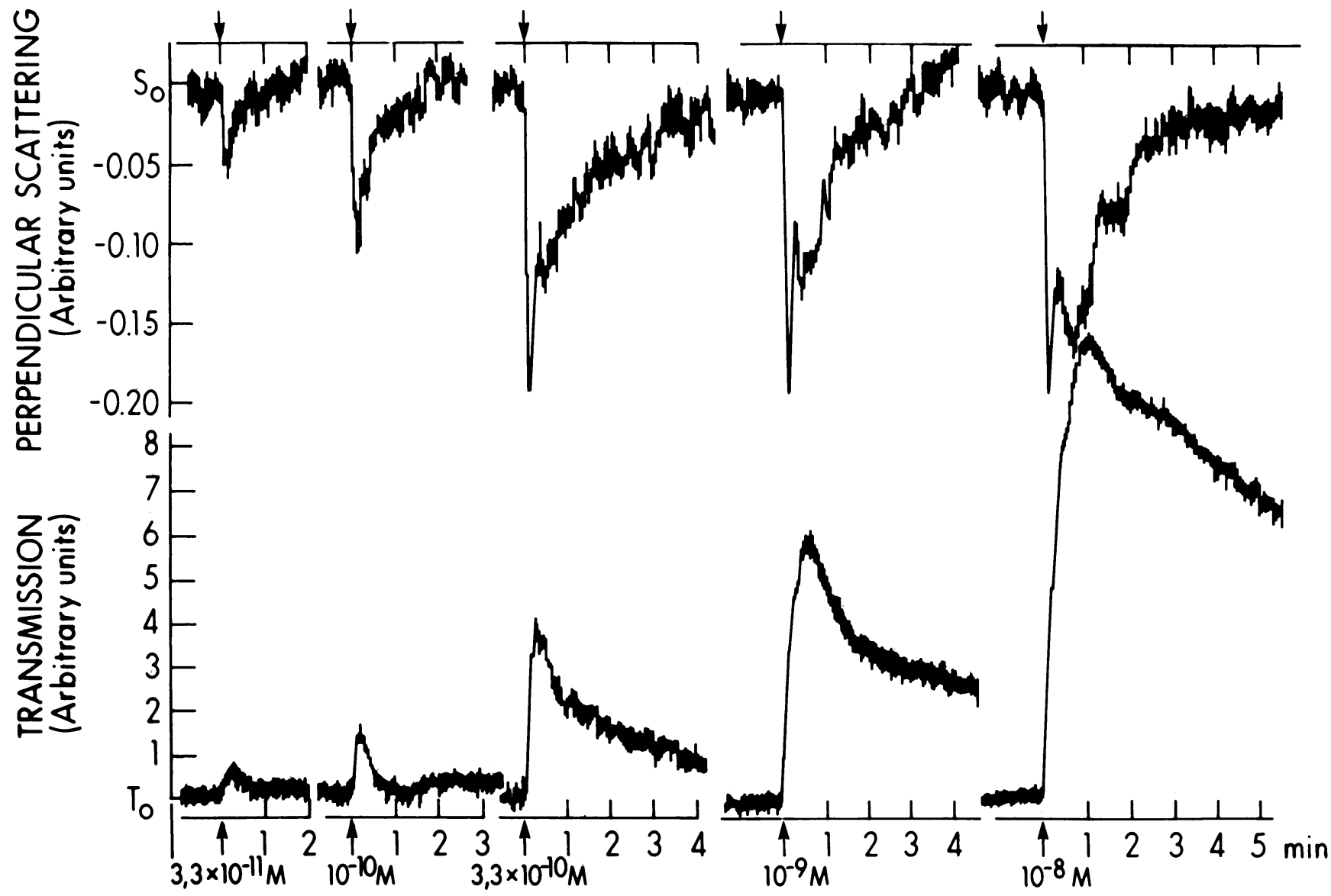

Figure 3. C5a dose-dependent induction of transmission and perpendicular scattering responses from PMN suspensions. The simultaneous measurements were performed using various concentrations of acid-purified C5a, which were added to the systems by 10- $\mu$ l injections at the indicated zero-time points.

the affinity of the PMN receptors for the chemoattractant as well as for the cells' chemotactic responsiveness, but markedly reduce their ability to secrete lysosomal enzymes or initiate a respiratory burst (16). Fig. 4 presents the $0.25 \%$ butanol effect on the light transmission response curves of PMN stimulated with fMet-Leu-Phe. These cells revealed a divergent light response exhibiting the fast peak response followed by a rapid drop in transmitted light intensity. At higher fMet-Leu-Phe doses the slowest peak was manifest, albeit at a low level. Similar effects were noted when PMN were treated with $0.1 \% \mathrm{~N}$-pentanol, a dose that produces equivalent effects on the above listed functions of the cells (data not shown). PMA, a potent inducer of $\mathrm{O}_{2}^{-}$production and lysosomal enzyme secretion, but not a chemoattractant, evoked no rapid response whatsoever, but initiated a slow response with lag-times of $\sim 1$ and $1.5 \mathrm{~min}$ at the forward and the perpendicular measurements, respectively (Fig. 5).

The role of cytoskeletal elements in light scattering by PMN. The role of cytoskeletal elements in producing the light changes by PMN was investigated by determining the transmission and

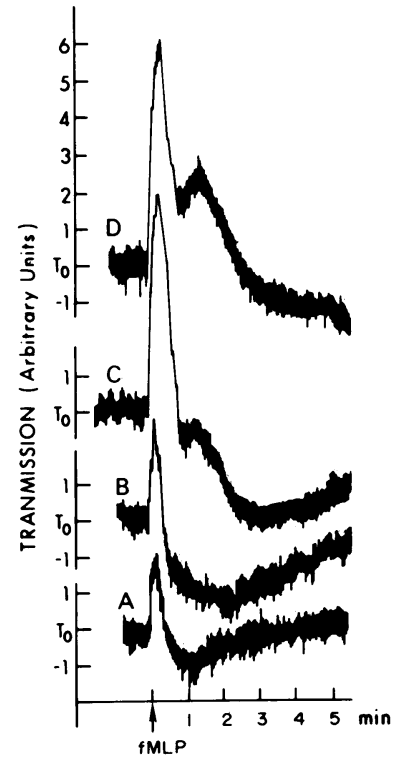

Figure 4. Effect of $N$-butanol on fMet-Leu-Phe dose-dependent transmission response from PMN suspensions. The cells were preincubated with $0.25 \% N$-butanol for $15 \mathrm{~min}$ at room temperature, then assayed in the presence of the alcohol. Stimulation was achieved with $0.1 \mathrm{nM}(A), 1 \mathrm{nM}(B), 10$ $\mathrm{nM}(C)$, and $100 \mathrm{nM}(D)$ fMetLeu-Phe that was added to the PMN suspensions at the indicated zero-time points. 


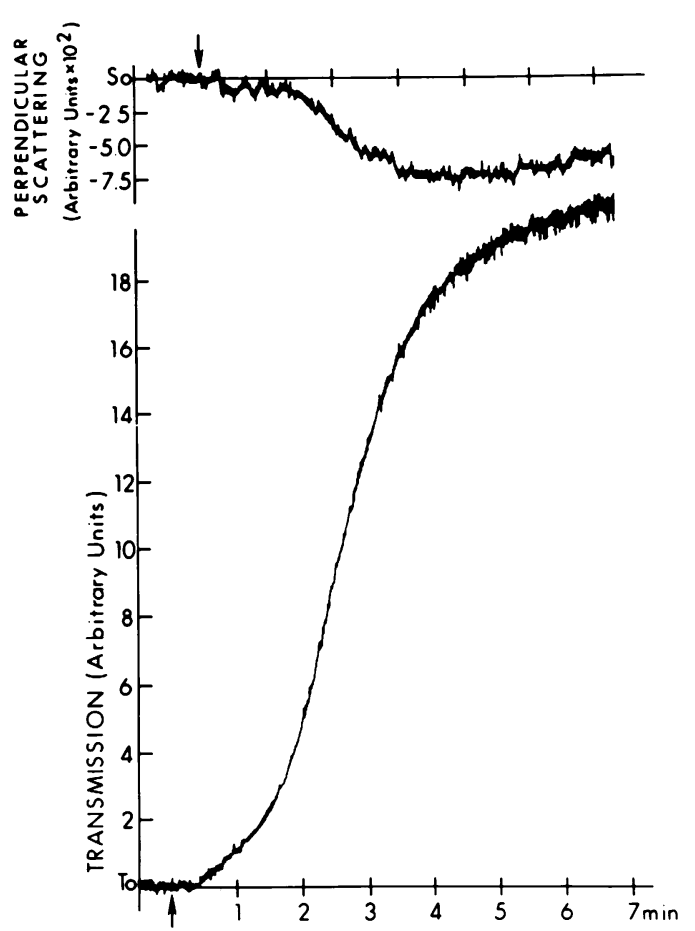

Figure 5. Simultaneous transmission and perpendicular scattering responses obtained from PMN suspension stimulated by PMA. The ligand was added to a final concentration of $10 \mathrm{nM}$ at the indicated zero-time point.

perpendicular light scattering in the presence of various cytoskeleton impairing agents. At concentrations up to $10 \mu \mathrm{M}$ neither colchicine, which disrupts the microtubule system, nor taxol, which prevents the depolymerization of microtubules (18) and consequently promotes their rate of assembly (19), affected the light intensity output of resting cells. These drugs did not interfere with the light-scattering responses induced by fMet-Leu-Phe (data not shown), either. In contrast, $1 \mu \mathrm{M} \mathrm{CB}$ abolished the rapid response to $10 \mathrm{nM}$ fMet-Leu-Phe. The slower responses were evoked with no detectable latency in freshly prepared PMN. The amplitudes of these responses revealed a shallow perpendicular scattering coupled with a prominent transmission response. In addition, CB markedly attenuated the relaxation of the slow response upon fMet-Leu-Phe stimulation (Fig. 6).

Morphological studies of PMN initiating light-scattering phenomena. In an attempt to identify the nature of the scattering source, PMN morphology was examined at the end of various periods of exposure to $10 \mathrm{nM}$ fMet-Leu-Phe. The morphological state was synchronized with the kinetics of the light-scattering response by rapidly fixing the same cell suspensions that had been monitored for their light responses to the chemoattractant. The various times of the cells' fixation are indicated by the termination of each light-scattering tracing presented in Fig. 7. Scanning electron micrographs were taken at low and high magnifications to allow examination of single cells as well as cell populations (Fig. 8). The low magnification photomicrographs demonstrate polarization of PMN by 2 min (Fig. $8 \mathrm{C}$ ), but tail to tail aggregation of the polarized PMN was documented only at $10 \mathrm{~min}$ (Fig. $8 \mathrm{D}$ ). The high magnification scanning electron micrographs demonstrate an evolution of the polarized PMN morphology, which is clear at the samples taken 2 and $10 \mathrm{~min}$ from stimulation. The kinetics of polarization and aggregation were confirmed by phase-light microscopy $(\times 400)$ using unfixed cells taken from the platelet aggregometer cuvette at the same times indicated in Figs. 7 and 8 (data not shown).

\section{Discussion}

The exposure of PMN to chemoattractants initiates the induction of a coordinated series of responses at the biochemical and cellular levels $(20,21)$. These responses are initiated by the binding of the chemoattractant to specific cell surface receptors (17, 22-27). The various responses mediated by the oligopeptide chemoattractant receptor on human PMN can be dissected into two major groups (28): those that occur at low chemoattractant doses, e.g., morphological polarization, chemokinesis, and che-

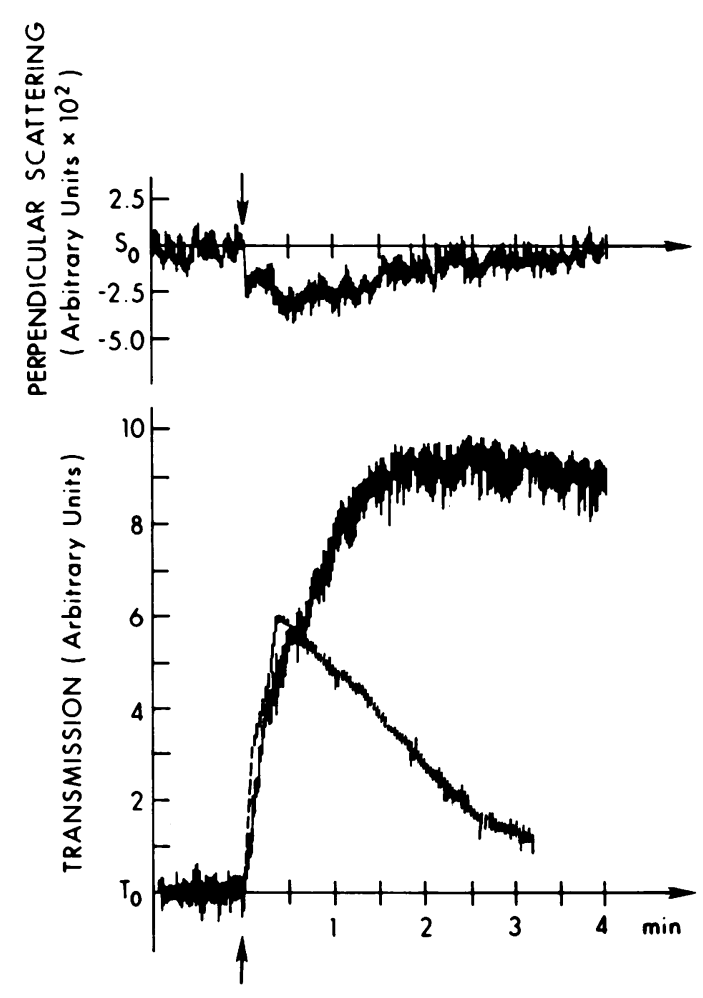

Figure 6. Effect of $\mathrm{CB}$ on the simultaneous transmission and perpendicular scattering responses of PMN suspensions to fMet-Leu-Phe. The cells were pretreated with $1 \mu \mathrm{M}$ CB for $15 \mathrm{~min}$ at room temperature, then stimulated with $10 \mathrm{nM}$ fMet-Leu-Phe at the indicated zero-time points. The dashed response represents control transmission in the absence of CB. 

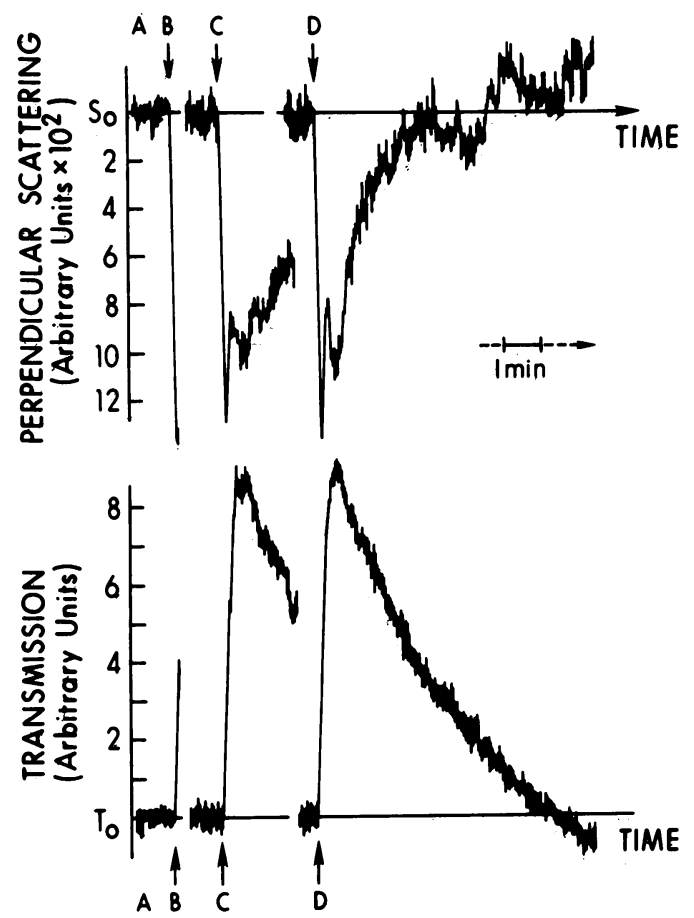

Figure 7. Arrested kinetics of simultaneausly recorded transmission and perpendicular scattering of PMN suspension responding to fMetLeu-Phe. The cell suspensions were stimulated with fMet-Leu-Phe at a final concentration of $10 \mathrm{nM}$ at the indicated zero-time points. The light responses were recorded for each experimental system until the addition of $0.4 \mathrm{ml}$ ice-cold $10 \%$ formaldehyde in HHB to the stirred cell suspension before $(A)$ or after $10 \mathrm{~s}(B), 2 \mathrm{~min}(C)$, and $10 \mathrm{~min}$ $(D)$ of stimulation, the timepoints at which the light tracings were interrupted due to the profound dilution effect.

motaxis (28), and others that require $\geq 10$-fold higher concentrations, e.g., lysosomal enzyme and superoxide anion secretion $(29,30)$. Recently, we observed that the two types of responses by PMN to fMet-Leu-Phe could be divergently altered by pharmacological means (28), suggesting that chemotactic and secretory functions are regulated differently.

Attempts to correlate dynamic cellular processes with receptor-binding characteristics measured under equilibrium or steady state conditions has a number of obvious drawbacks, not the least of which is that many responses are complete at low receptor occupancy $(31,32)$ before equilibrium of receptor occupancy is reached. For these reasons, the understanding of a chemoattractant's interaction-induction mechanism requires a kinetic approach with an emphasis on short time scales. Practically, it implies the need for a correlated study of the chemoattractants' association with a fast response, preferably in a continuous assay. This approach has already been used for responses induced by the high dose range of chemoattractants and measured in the continuous assays of $\mathrm{O}_{2}^{-}$production (33), oxygen consumption (34), lysosomal enzyme secretion $(35,36)$, and the modulation of the PMN membrane potential $(37,38)$.
A common feature of these responses is a response lag time, which varies among the assays with respect to the different chemoattractants and their doses. This lag time indicates that the responses are either of a complex reaction order or successive to other responses. As yet, no real-time approach has been successfully ascribed to the motility-related responses of PMN that are induced by the low dose range of the chemoattractants. Accordingly, we attempted to monitor the morphological po-
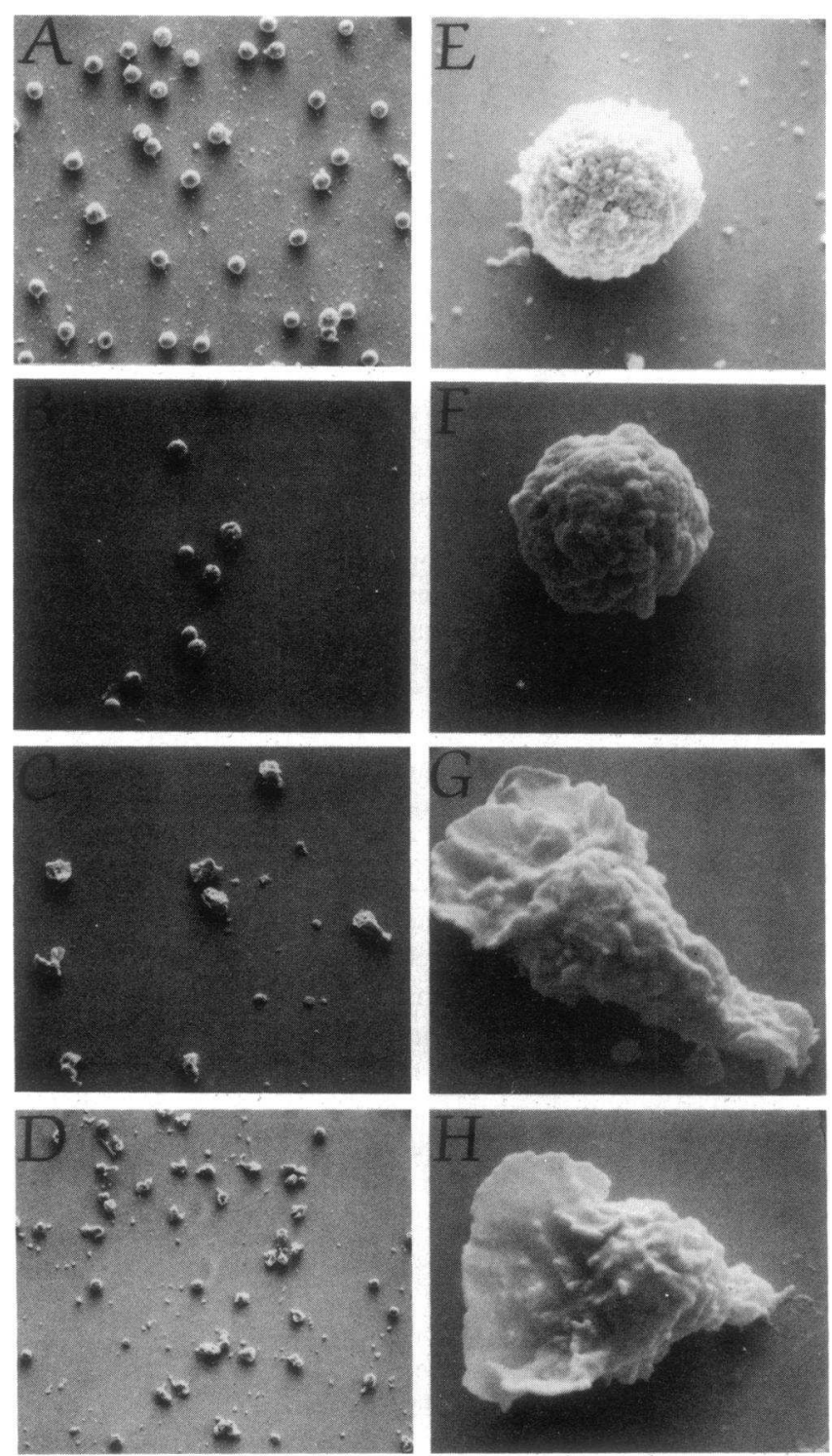

Figure 8. Scanning electron micrographs of the PMN fixed in the arrested kinetic experiments reported on Fig. 7. $A-D$ (drawing upon the same notations of Fig. 7) demonstrate arbitrarily chosen PMN populations, presented at $\times 600(A-C)$ and $\times 300(D)$ magnification. $E-H$ each present a single cell chosen from $A-D$, respectively, at $\times$ 6,000 magnification. 
larization of PMN, to the shape characteristic of migratory cells. To do this, we monitored the initial phase of alterations in PMN light transmission profiles in a platelet aggregation meter after the introduction of chemoattractants. The addition of the perpendicular scattering measurement enabled us to refine the understanding of the light modulation response that occurs subsequent to the PMN interaction with a chemoattractant.

Changes in light absorption by PMN did not appear to interfere in the measurements, as evidenced by the general reciprocal changes in the intensities of the simultaneously monitored transmission and the perpendicular scattering. If absorption occurred it would have resulted in an angular independent and proportional decrease of light intensity in both the transmission and the perpendicular scattering. Thus, the light intensity alterations from PMN suspensions result primarily from modulation of light scattering. The opposing direction of the lightscattering responses at the 0 and $90^{\circ}$ indicate that the light intensity emanate from morphological changes rather than from biochemical reactions. The latter would modify the cells' scattering power by an even scaling effect throughout the angular profile of the scattered light. A scaling effect would consequently appear as a unidirectional and proportional change in the light intensities at any given pair of observation angles. The measurements carried out at various PMN densities enabled the identification of the multiple-scattering interference, which indeed resulted in a nonlinear optical density pattern, and a nonquantitative response to cell concentration in the transmission mode. However, the perpendicular observation, which is far less affected by multiple scattering, revealed a sufficiently linear dependence on cell concentration to be an acceptable quantitative measure. Measurements of perpendicular scattering also permitted the clear identification of two time-resolved light modulation responses, which were only suggested by the transmission data. This was the result of the better resolution (i.e., significance over base-line level) and the higher synchronization of the rapid response in the perpendicular measurement.

Simultaneous measurement of transmission and perpendicular light-scattering responses by PMN indicate that the rapid and slow responses are regulated differently in regards to chemoattractant dose, membrane fluidity, and cytoskeletal requisites. The half-maximal effective concentrations of chemoattractant for the rapid and the slow responses were distinct by an order of magnitude, as the rapid response was lower than the slow one. This distinction held true for all chemoattractants tested, and corresponded closely to their chemoattractant-receptor affinities and $\mathrm{EC}_{50}$ for the induction of PMN migratory and secretory responses. The rapid and slow responses could be further distinguished by the effects of aliphatic alcohols. Butanol at doses previously shown to enhance chemotactic responsiveness but to inhibit secretory functions of PMN (16) did not depress the rapid response, but virtually obliterated the slower response at all but the highest doses of chemoattractant. CB, which inhibits cell motility but enhances secretion (39-41), entirely abrogated the rapid but not the slow response to fMet-
Leu-Phe. However, in this case, or with PMA as a stimulant, a discrepancy was noted between the ratio of transmission to perpendicular scattering amplitudes of the slow responses when they are compared with the same ratio in PMN responding to fMet-Leu-Phe alone. Moreover, the transmission response in the former two instances demonstrate a sustained near-maximum elevation. The explanation for these observations is not yet clear, however a hypothesis can be raised that is based on previous observations that fMet-Leu-Phe plus CB or PMA, but not fMet-Leu-Phe alone, cause extensive lysosomal enzyme secretion by PMN. This secretion should decrease the PMN average refractive index due to the discharge of dense lysosomal granules. Assuming an overall decrease of the cells' refractive index with respect to the medium, a scaling down of the scattering power and an increased transmission are expected. Secretion could therefore account for the apparent lack of relaxation of the slow transmission response in PMA or fMet-Leu-Phe plus CB-treated cells due to an up-shift of the transmission base-line level.

We attempted to identify the origin of the light-scattering changes by a morphological approach. Taken from platelet aggregation, it has generally been deduced that the light transmission increase seen in PMN suspensions exposed to chemoattractants was due to an aggregation process. This contention was made plausible by the observations that PMN aggregation does indeed occur $\sim 1-4$ min $(7,8)$ after the introduction of a chemoattractant. The aggregation analogy was further strengthened by the transmission profiles obtained in many studies where $\mathrm{CB}$ was used, and therefore prevented decay of the transmission peak $(13,42-47)$. Our morphological data indicate that while aggregation occurs sometime after 2 min of the introduction of the chemoattractant, it cannot account for the major portion of the changes in light scattering that reaches maximal amplitudes within the first minute after the chemoattractant is added. Furthermore, the morphological polarization of PMN did not become apparent by phase-light microscopic examination of viable cells until $\sim 30 \mathrm{~s}$. In the present study, polarization was fully developed by $2 \mathrm{~min}$ and persisted to at least $10 \mathrm{~min}$. Polarization then could not account for the rapid perpendicular light-scattering phenomenon, which was essentially complete by $20 \mathrm{~s}$. Neither could polarization account for the slower response peak, since polarization does not occur in the presence of $\mathrm{CB},(6)$ and in the absence of $\mathrm{CB}$ the slow response declined to base line within $10 \mathrm{~min}$ at doses of fMet-Leu-Phe that caused sustained polarization of the PMN. It can thus be concluded that while chemoattractants induce PMN aggregation and polarization, these phenomena are not major contributors to the light transmission alterations. This leads, by logical induction, to the notion that the light-scattering source may be of subcellular dimensions. This notion is circumstantially supported by the relatively small amplitudes of the light responses and the lack of angular dependence at the higher observation angles. In addition to the above, the previously reported rates of pseudopodia formation by PMN in response to chemoattractants (48), and the relation 
of the light responses to the cytoskeleton-disrupting agents, led us to propose membrane ruffling and/or intracellular granules as the scattering objects. The former would be assumed to rapidly and reversibly change their size, shape, or arrangement on the surface of PMN exposed to even low doses of chemoattractants. The latter would be assumed to change their position, i.e., their spacial distribution, or refractive index with respect to the cells' cytoplasm. Morphological approaches taken thus far have not resolved the scattering source perhaps due to limits of resolution (i.e., size or contrast). Complex physical measurements, such as angular dependence of the scattered light over a wide angle range $\left(0^{\circ} \leq \theta \leq 180^{\circ}\right)$ and determination of index or refraction from locations within single cells, could provide additional information concerning the size and nature of the source of scattering.

The identification of the rapid perpendicular scattering response of PMN is of considerable interest. The instantaneous development, short life-time, low $\mathrm{EC}_{50}$ and pharmacological characteristics of this response qualify it as an early event in the induction of PMN chemotaxis. Based on the nature of the experimental system, which does not maintain any spacial gradient, this response becomes the first defined measure of the PMN perception of a temporal chemoattractant gradient (49).

\section{Acknowledgments}

We are deeply thankful to Mr. S. Simons (Sienco, Inc.) for the elegant modification of the Sienco aggregation meter to meet the requirements of this work, Drs. J. Vergara and P. Ingram for the preparation and performance of the scanning electron micrographs, Dr. B. Lane for the donation of the C5a fraction, Dr. W. Longley for his interest in this work and invaluable help in the interpretation of the light scattering, Dr. I. Fridovich for his very helpful comments on the manuscript, and Ms. J. Telander and Ms. Sharon Goodwin for their secretarial assistance.

\section{References}

1. Koshland, D. E., Jr. 1980. In Bacterial Chemotaxis as a Model Behavioral System. Raven Press, New York. 27-32, 55-68.

2. Zigmond, S. H. 1977. Ability of polymorphonuclear leukocytes to orient in gradients of chemotactic factors. J. Cell Biol. 75:606-616.

3. Miller, M. E. 1979. Cell elastimetry in the study of normal and abnormal movement of human neutrophils. Clin. Immunol. Immunopathol. 14:503-510.

4. Smith, C. W., J. C. Hollers, R. A. Patrick, and C. Hassett. 1979. Motility and adhesiveness in human neutrophils. Effect of chemotactic factors. J. Clin. Invest. 63:221-229.

5. Kawaoka, E. J., M. E. Miller, and A. T. W. Cheung. 1981. Chemotactic factor-induced effects upon deformability of human polymorphonuclear leukocytes. J. Clin. Immunol. 1:41-44.

6. Cianciolo, G. J., and R. Snyderman. 1981. Monocyte responsiveness to chemotactic stimuli is a property of subpopulation of cells that can respond to multiple chemoattractants. J. Clin. Invest. 67:60-68.

7. Craddock, P. R., D. Hammerschmidt, J. G. White, A. P. Dalmasso, and H. S. Jacob. 1977. Complement (C5a)-induced granulocyte aggregation in vitro. A possible mechanism of complement-mediated leukostasis and leukopenia. J. Clin. Invest. 60:260-264.
8. Hoffstein, S. T., R. S. Friedman, and G. Weissmann. 1982. Degranulation, membrane addition and shape change during chemotactic factor-induced aggregation of human neutrophils. J. Cell Biol. 95:234241.

9. Jacob, H. S., and D. Hammerschmidt. 1981. Complement-induced granulocyte aggregation. Importance in myocardial infarction and shock lung. J. Am. Med. Assoc. 245:2013-2017.

10. MacMillan, D. C., and M. R. Oliver. 1965. The initial change in platelet morphology following the addition of adenosine dephosphate. J. Atheroscler. Res. 5:440-444.

11. O'Brien, J. R., and M. A. Wodehouse. 1968. Platelets: their size, shape and stickiness in vitro; degranulation and propinquity. Exp. Biol. Med. 3:90-102.

12. Oseas, R. S., L. A. Boxer, C. Butterick, and R. L. Baehner. 1980. Differences in polymorphonuclear leukocyte aggregating responses among several species in response to chemotactic stimulation. J. Lab. Clin. Med. 96:213-221.

13. Yasaka, T., L. A. Boxer, and R. L. Baehner. 1982. Monocyte aggregation and superoxide anion release in response to formyl-methionyl-leucyl-phenylalanine(FMLP) and platelet-activating-factor(PAF). J. Immunol. 128:1939-1944.

14. Boyum, A. 1968. Isolation of mononuclear cells and granulocytes from human blood. Isolation of mononuclear cells by one centrifugation and of granulocytes by combining centrifugation and sedimentation at 1 g. Scand. J. Clin. Lab. Invest. 21(Suppl. 97):77-89.

15. Cohen, A. L. 1974. In Principles and Techniques of Scanning Electron Microscopy, Vol. 1, M. A. Hayat, editor. Van Nostrand Reinhold Co. Inc., New York. 44-112.

16. Yuli, I., A. Tomonaga, and R. Snyderman. 1982. Chemoattractant receptor functions in human polymorphonuclear leukocytes are divergently altered by membrane fluidizers. Proc. Natl. Acad. Sci. USA. 79:5906-5910.

17. Niedel, J., S. Wilkinson, and P. Cuatrecasas. 1979. Receptormediated uptake and degradation of ${ }^{125}$ I-chemotactic peptide by human neutrophils. J. Biol. Chem. 254:10700-10706.

18. Pasness, J., and S. B. Horwitz. 1981. Taxol binds to polymerized tubulin in vitro. J. Cell Biol. 91:479-487.

19. Autin, P. B., S. Forry-Schandies, T. M. Friedman, S. J. Tapscott, and $\mathrm{H}$. Holtzer. 1981. Taxol induces postmitotic myoblast to assemble interdigitating microtubule-myosin assays that exude actin filament. $J$. Cell Biol. 90:300-308.

20. Snyderman, R., I. Yuli, and C. Koo. 1983. Pharmacological manipulation of the chemotactic factor receptor on leukocytes. In Biological Response Mediators and Modulators. Academic Press, Inc. New York. 211-219.

21. Snyderman, R., and E. J. Goetzl. 1981. Molecular and cellular mechanisms of leukocyte chemotaxis. Science (Wash. DC). 213:830837.

22. Aswanikumar, S., A. B. Corcoran, E. Schiffmann, A. L. Day, R. J. Freer, H. J. Showell, E. L. Becker, and C. B. Pert. 1977. Demonstration of a receptor on rabbit neutrophils for chemotactic peptides. Biochem. Biophys. Res. Commun. 74:810-818.

23. Williams, L. T., R. Snyderman, M. C. Pike, and R. J. Lefkowitz. 1977. Specific receptor sites for chemotactic peptides on human polymorphonuclear leukocytes. Proc. Natl. Acad. Sci. USA. 74:1204-1208.

24. Chenoweth, D. E., and T. E. Hugli. 1978. Demonstration of specific C5a receptor on intact polymorphonuclear leukocytes. Proc. Natl. Acad. Sci. USA. 75:3943-3947.

25. Spilberg, I., and J. Mehta. 1979. Demonstration of a specific 
neutrophil receptor for a cell-derived chemotactic factor. J. Clin. Invest. 63:85-88.

26. Pike, M. C., and R. Snyderman. 1982. Chemoattractant-receptor interactions in leukocytes. In Advances in Inflammation Research. G. Weissman, editor. Raven Press, New York. 109-129.

27. Goldman, D. W., and E. J. Goetzl. 1982. Specific binding of leukotriene B4 to receptors on human polymorphonuclear leukocytes. J. Immunol. 129:1600-1604.

28. Snyderman, R. 1984. Regulatory mechanisms of a chemoattractant receptor on leukocytes: chemotactic and secretory signals are independently transduced. Fed. Proc. In press.

29. Showell, H. J., R. J. Freer, S. H. Zigmond, E. Schiffmann, S. Aswanikumar, B. A. Corcoran, and E. L. Becker. 1976. The structureactivity relation of synthetic peptides as chemotactic factors and inducers of lysosomal enzyme secretion for neutrophils. J. Exp. Med. 143:11541169.

30. Lehmeyer, J. E., R. Snyderman, and R. B. Johnston. 1979. Stimulation of neutrophil oxidative metabolism by chemotactic peptides: influence of calcium ion concentration and cytochalasin B and comparison with stimulation by phorbol myristate acetate. Blood. 54:35-45.

31. Sklar, L. A., Z. G. Oades, A. J. Jesaitis, R. G. Painter, and C. G. Cochrane. 1981. Fluoresceinated chemotactic peptide and high affinity antibody to fluorescein as a probe of the temporal characteristics of neutrophil stimulation. Proc. Natl. Acad. Sci. USA. 78:7540-7544.

32. Sklar, L. A., A. J. Jesaitis, R. G. Painter, and C. G. Cochrane. 1983. Quantitative analysis of the relationship between receptor occupancy and cellular response in human neutrophil. Biophys. J. 41:132a. (Abstr.)

33. Smolen, J. E., H. M. Korchak, and G. Weissmann. 1980. Initial kinetics of lysosomal enzyme secretion and superoxide anion generation by human polymorphonuclear leukocytes. Inflammation. 4:145-163.

34. Alobaidi, T., P. H. Naccache, and R. I. Sha'afi. 1981. Calmodulin antagonist modulates rabbit neutrophil degranulation, aggregation and stimulated oxygen consumption. Biochim. Biophys. Acta. 675:316-321.

35. Smolen, J. E., H. M. Korchak, and G. Weissmann. 1982. The kinetics of lysosomal degranulation of human neutrophils as measured by $\alpha$-amino acridine self-quenching. J. Cell Biol. 95:397a. (Abstr.)

36. Brentwood, B. J., and P. M. Henson. 1980. The sequential release of granule constituents from human neutrophils. J. Immunol. 124:855862.

37. Seligmann, B., T. M. Chussed, and J. I. Gallin. 1981. Human neutrophil heterogeneity identified using flow microfluorometry to monitor membrane potential. J. Clin. Invest. 68:1125-1131.

38. Seligmann, B. E., M. P. Fletcher, and J. I. Gallin. 1982. Adaptation of human neutrophil responsiveness to the chemoattractant $N$-formylmethionyl-leucyl-phenylalanine. J. Biol. Chem. 257:6280-6286.

39. Goldstein, I., S. Hoffstein, J. Gallin, and G. Weissmann. 1973. Mechanism of lysosomal enzyme release from human leukocytes: microtubule assembly and membrane fusion induced by a component of complement. Proc. Natl. Acad. Sci. USA. 70:2916-2920.

40. Becker, E. L., and H. J. Showell. 1974. The ability of chemotactic factors to induce lysosomal enzyme release. II. The mechanism of release. J. Immunol. 112:2055-2062.

41. Bennett, J. P., S. Cockcroft, and B. D. Gomperts. 1980. Use of cytochalasin $B$ to distinguish between early and late events in neutrophil activation. Biochim. Biophys. Acta. 601:584-591.

42. Hammerschmidt, D., J. G. White, P. R. Craddock, and H. S. Jacob. 1979. Corticosteroids inhibit complement-mediated granulocyte aggregation. A possible mechanism for their efficiency in shock states. J. Clin. Invest. 63:798-803.

43. Hammerschmidt, D., T. K. Bowers, C. J. Lammi-Keefe, H. S Jacob, and P. R. Craddock. 1980. Granulocyte aggregometry: a sensitive technique for the detection of C5a and complement activation. Blood. 55:898-902.

44. Mease, A. D., D. P. Burgess, and P. J. Thomas. 1981. Irreversible neutrophil aggregation. A mechanism of decreased newborn neutrophil chemotactic response. Am. J. Pathol. 104:98-102.

45. Kraut, E. H., M. Segal, and A. L. Sagone, Jr. 1982. Evaluation of the role of oxygen radicals in polymorphonuclear leukocyte aggregation. Inflammation. 6:161-167.

46. Oseas, R. S., J. Allen, H. H. Yang, R. L. Baehner, and L. A. Boxer. 1982. Mechanism of dexamethasone inhibition of chemotactic factor-induced granulocyte aggregation. Blood. 59:265-269.

47. Redl, H., D. Hammerschmidt, and G. Schlag. 1983. Augmentation by platelets of granulocytes aggregation in response to chemotaxis: studies utilizing an improved cell preparation technique. Blood. 61:125131.

48. Zigmond, S. H., and S. J. Sullivan. 1979. Sensory adaptation of leukocytes to chemotactic peptides. J. Cell Biol. 82:517-527.

49. Yuli, I., and R. Snyderman. 1983. Rapid perpendicular light scattering(LS): a previously unrecognized response of human neutrophils(PMNs) to chemoattractants(CTX). Clin. Res. 31:379a. (Abstr.) 